\title{
REFERATENTEIL.
}

\section{ERGEBNISSE DER NEUEREN ARBEITEN ÜBER DEN CHEMISMUS DER ALKOHOLISCHEN ZUCKER- SPALTUNG.}

\author{
Von \\ Julius Hirsch (Berlin-Dahlem).
}

Die nenere Literatur, die sich mit den Untersuchungen über die Funktionen der Hefezellen beschäftigt, breitet sich über ein Gebiet aus, das fast alle Fragen der Biochemie und der Biophysik berührt. Neben der eigentlichen Gärungschemie ist es vornehmlich die Fermentforschung, die sich schon wegen der leichten Zugänglichkeit und der bequemen Handhabung des Materials gern der Hefezelle bedient. Hierher gehört eine große Anzahl von Arbeiten, die die Reindarstellung von Fermenten, sowie die Aufklärung der Kinetik fermentativer Vorgänge zum Ziele haben. Sodann sind viele Probleme, die die physikalische Chemie der Zelle betreffen, an der Hefezelle untersucht worden. Uber die Ergebnisse all dieser Arbeiten zu berichten, dürfte nicht nur die räumlichen Grenzen eines Referates übersteigen, sondern müßte wegen der Heterogenität der Problemstellungen einer gewissen Ubersichtlichkeit entbehren. Es soll daher im folgenden nur von den Arbeiten die Rede sein, die sich mit der chemischen Seite der alkoholischen Zuckerspaltung als der sinnfälligsten Funktion der lebenden Hefe und der Hefefermente befassen.

Die Fähigkeit der Hefezelle, aus Zucker Alkohol zu produzieren, ist seit altersher bekannt, und schon vor roo Jahren konnte GAYLussac den Beweis erbringen, daß hierbei ein Molekül Hexose in zwei Moleküle Äthylalkohol und zwei Moleküle Kohlendioxyd zerfällt: $\mathrm{C}_{6} \mathrm{H}_{12} \mathrm{O}_{6}=2 \mathrm{C}_{2} \mathrm{H}_{5} \mathrm{OH}+2 \mathrm{CO}_{2}$. Mit dieser Beobachtung waren die Endprodukte des Kohlenhydratstoffwechsels der Hefezelle quantitativ analysiert und es hat lange Zeit gedauert, bis die experimentelle Forschung die genetische Aufklärung der rein stöchiometrischen Befunde GAY-Lussacs in Angriff nahm. Während die Frage nach der Fermentnatur der Gärung durch die Arbeiten von Pasteur, Liebig, sowie von $E$. und $H$. Buchner und $M$. HAHN behandelt wurde, blieben die chemischen Probleme hypothetischen Spekulationen überlassen, die ohne hinreichende experimentelle Begründung die verschiedensten Substanzen als Zwischenprodukte bei dem Kohlenhydratabbau durch die Hefezelle bzw. das Hefeferment bezeichneten.

Zum Beginn des Jahres Igr I berichtete Neuberg, daß in ausgesprochener Analogie zur Vergärung des Zuckers die Brenztraubensäure durch Hefe und Hefeferment unter Entwicklung von Kohlensäure aufgespaiten wird, wobei anstatt des Athylalkohols Acetaldehyd entsteht. Mit dieser Beobachtung setzte die neuere Gärungschemie ein, deren Hauptergebnisse in NeUbERGs Laboratorium gezeitigt wurden.

Die glatte Vergärbarkeit der Brenztraubensäure, die sich praktisch unter denselben Bedingungen wie die der Hexosen vollzieht, berechtigte schon an sich zu der Annahme, daß dieser 3-Kohlenstoffkörper ein wesentliches $Z$ wischenprodukt des fermentativen Abbaues der Kohlenhydrate bildet, zumal sie nicht nur von der Hefezelle, sondern von allen zymasehaltigen Organismen angegriffen wird und somit das Brenztraubensäure spaltende Ferment die Carboxylase (so genannt, weil sie die Carboxylgruppe abspaltet)als ein Teilferment der Zymase anzusprechen ist. Weiterhin mußte logischerweise auch der aus der Brenztraubensäure gemäß der Gleichung: $\mathrm{CH}_{3} \mathrm{CO} \cdot \mathrm{COOH}=\mathrm{CH}_{3} \mathrm{CHO}+\mathrm{CO}_{2}$ entstehende Acetaldehyd ein intermediäres Glied des Zuckerzerfalls darstellen. und es gelang in der Tat, sowohl den Acetaldehyd wie auch die Brenztraubensäure durch Fixierung mittels gewisser Abfangverfahren aus dem intermediären Prozesse herauszugreifen (s. unten).

Die theoretischen Vorstellungen von den Teilvorgängen beim Zerfall des Zuckermoleküls in Alkohol und Kohlensäure formulierte NEUBERG (19I3) in nebenstehendem Schema:

Die Anfstellung dieses Schemas geschah auf Grund der experimentell nachgewiesenen Vergärbarkeit der Brenztraubensäure sowie unter Berücksichtigung bestimmter rein chemischer Er- fahrungen. Die Arbeiten der folgenden Jahre haben in fast lückenloser Beweisführung gezeigt, daß die neue Gärungstheorie dem tatsächlichen Geschehen weitgehend entspricht. Zum besseren Verständnis werde ich bei der Besprechung dieser Theorie die bestätigenden experimentellen Befunde ohne Rücksicht auf ihre zeitliche Reihenfolge heranziehen.

Die Gleichung $\alpha$ obiger Formelbilder besagt, da $\beta$ unter dem Einflusse des Hefefermentes dem Hexosemólekül 2 Moleküle Wasser entzogen werden, wobei 2 Moleküle des 3 -Kohlenstoffkörpers Methylglyoxal entstehen. Die Möglichkeit eines derartigen Zerfalles ergab sich aus der rein chemischen Beobachtung, daß Zucker nicht nur bei der Behandiung mit starker Kalilauge, sondern auch mit schwach alkalisch reagierenden Salzen - wie mit Soda, Natriumbikarbonat, Phosphaten, Sulfiten, Borax und Natriumacetat (NeUBerg, Oerrel u. Rewatd) - Methylglyoxal liefern kann. Es wird zwar das auf rein chemischen Wege darstellbare Methylglyoxal von lebender Hefe nicht vergoren, doch besagt dies nichts, wenn man bedenkt, daß eine große $\mathrm{Zahl}$ isomerer (auch optisch-aktiver) Formen des Methylglyoxal möglich ist, deren chemische Präparation bisher noch nicht gelang. Der an die Aufspaltung der Hexose in 2 Moleküle Methylglyoxal sich anschließende Vorgang $\beta$ fordert eine Cannizzarosche Reaktion zwischen einem Molekül Methylglyoxal und einem Molekül Methylglyoxalhydrat. Unter Aufnahme eines Moleküls Wasser wird ein Molekül Glycerin und ein Molekül Brenztraubensäure gebildet. Die Cannizzarosche Reaktion ist ein in der Natur weit verbreiteter fermentativer Vorgang. Die Aldehydmutase, die sowohl in tierischen, wie pflanzlichen Zellen, insbesondere auch in der Hefe'nachgewiesen worden ist, wandelt zwei Moleküle Aldehyd zu einem Molekïl Alkohol und zu einem Molekül Säure um, indem sie ein Molekül Wasser aufnehmen läßt. Gibt man z. B. zu einer Suspension von Hefezellen Acetaldehyd, so entstehen Äthylalkohol und Essigsäure in molekularem Verhältnis (Nevberg u. KERB, Kostytschew). Die optimale Wirksamkeit der Aldehydmutase liegt bei schwach alkalischer Reaktion (Neuberg u. Hirsch), doch ist die Dismutation von Aldehyden auch bei der H-Ionenkonzentration normaler Gäransätze in beträchtlichem Umfange nachzuweisen. Die Cannizzarosche Reaktion ist somit als ein Teilvorgang der Zymasewirkung anzusprechen und sind die durch Dismutation aus dem Methylglyoxal entstehenden Produkte Glycerin und Brenztraubensäure tatsächlich nachzuweisen.

Der Umfang, in welchem sich die Gleichung $\beta$ während des Gäraktes vollzieht, ist nur gering. Die Menge des Glycerins, das im weiteren Verlaufe der Umsetzungen unangegriffen bleibt und analytisch nachweisbar ist, beträgt maximal $3 \%$ des Ausgangsmaterials, wobei wir jedoch noch unberücksichtigt lassen, daß außerdem ein anderer Entstehungsmodus (s. unten) für das Glycerin hinzukommen kann. DaB unter den Endprodukten normalerweise nicht auch Brenztraubensäure nachzuweisen ist, ergibt sich aus ihrer leichten Vergärbarkeit; sie zerfällt in statu nascendi in Acetaldehyd und Kohlensäure gemäß Gleichung $\gamma$, die somit experimentell völlig verifiziert werden konnte. Der aus der Brenztraubensäure abgespaltene Acetaldehyd unterliegt sodann nach Formelbild $\delta$ zusammen mit einem Molekül Methylglyoxal der Cannizzaroschen Reaktion. Die aus dieser, gekreuzten Dismutation" zweier verschiedener Aldehyde entstehende Säure ist wiederum Brenztraubensäure und der entsprechende Alkohol der als Gärungsendprodukt auftretende Äthylalkohol. Dieser reichert sich an, während die Brenztraubensäure stets unter Entwicklung freier Gärungskohlensäure decarboxyliert wird $(\gamma)$, um dem nach $\alpha$ gebildeten Methylglyoxal als Dismutationspaarling Acetaldehyd zu liefern. Die Möglichkeit der

$$
\begin{aligned}
& \text { a) } \mathrm{C}_{6} \mathrm{H}_{12} \mathrm{O}_{6}-2 \mathrm{H}_{2} \mathrm{O}=2 \mathrm{CH}_{3} \cdot \mathrm{CO} \cdot \mathrm{COH} \text { (Methylglyoxal), } \\
& \text { B) } \mathrm{CH}_{2}: \mathrm{C}(\mathrm{OH}) \cdot \mathrm{COH}_{2}+\mathrm{H}_{2} \mathrm{O} \quad \mathrm{H}_{2} \quad \mathrm{CH}_{2} \mathrm{OH} \cdot \mathrm{CHOH} \cdot \mathrm{CH}_{2} \mathrm{OH} \text { (Glyzerin) }
\end{aligned}
$$

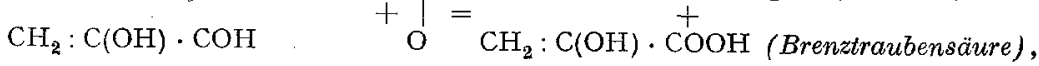

$$
\begin{aligned}
& \text { y) } \mathrm{CH}_{3} \cdot \mathrm{CO} \cdot \mathrm{COOH} \quad=\mathrm{CO}_{2}+\mathrm{CH}_{3} \cdot \mathrm{COH} \text { (Acetaldehyd), } \\
& \text { d) } \mathrm{CH}_{3} \cdot \mathrm{CO} \cdot \mathrm{COH} \quad \mathrm{O} \quad \mathrm{CH}_{3} \cdot \mathrm{CO} \cdot \mathrm{COOH} \text { (Brenztraubensäure) } \\
& \mathrm{CH}_{3} \cdot \mathrm{COH}+\mathrm{H}_{2}=\mathrm{CH}_{3} \cdot \mathrm{CH}_{2}^{+} \mathrm{OH} \text { (Alkohol). }
\end{aligned}
$$


gekreuzten Cannizzaroschen Reaktion ist durch einen chemischen Modellversuch erwiesen, bei dem unter dem Einflusse chemischer Reagenzien aus Acetaldehyd und Benzaldehyd nur Essigsä ure und Benzylalkohol erhalten wurde (NORD).

Die Darstellung der intermediär auftretenden und gleich verschwindenden Brenztraubensäure wurde durch eines der so bedeutsam gewordenen Abfangverfahren ermöglicht. Nach DöBNER verbinden sich ein Molekül $\beta$-Naphtylamin mit 2 Molekülen Brenztraubensäure unschwer zur 3-Methyl- $\beta$-naphtocinchoninsäure. Die Vergärung von Zucker durch Hefefermentsaft in Gegenwart von $\beta$-Naphtylamin führte zu einer Fixierung der Brenztraubensäure in Form des erwähnten Kondensationsproduktes (v. GraB). Nach anderen Abfangprinzipien, über die sogleich ausführlich berichtet werden $\mathrm{muB}$, war schon vorher der Acetaldehyd vor der weiteren Umsetzung bewahrt worden, so daß die Richtigkeit der entwickelten Theorie durch die Isolierung wesentlicher Zwischenprodukte und die experimentelle Darstellung wichtiger Teilvorgänge weitgehend erwiesen worden ist.

Die Ausschaltung des Acetaldehyds aus der gewöhnlichen Reaktionsfolge war durch die Anwendung der Sulfitmethode (NeUBerg und REINFURTH) in einem Umfange möglich, der infolge seines Ausmaßes einen genauen Einblick in das feinere Getriebe des Gärungsmechanismus gestattete. Läßt man Zucker in Gegenwart von alkalischem schwefligsaurem Natrium (oder Calcium) vergären, so bindet dieses Carbonylreagenz den gemäß Gleichung $\gamma$ intermediär auftretenden Acetaldehyd in Form des Acetaldehydnatriumbisulfites und bewahrt ihn vor der weiteren Hydrierung zu Äthylalkohol. Auf diese Weise konnte man bedeutende Mengen dieses $Z$ wischenproduktes anreichern und durch Anwendung der üblichen Trennungsmethoden aus dem Sulfitkomplex in reiner Form präparativ darstellen. Bei Verwendung maximal ertragener Mengen von Abfangmittel wurden bis $75 \%$ des nach der Theorie möglichen Acetaldehyds festgelegt. Durch die Fixierung wurde der Acetaldehyd der weiteren Reduktion zu Äthylalkohol (Gleichung $\delta$ ) entzogen, und es war zu erwarten, daß der hierdurch verfügbar werdende Wasserstoff entweder als solcher mit den Gärungsgasen entweicht oder in Form eines der Acetaldehydmenge äquivalenten Reduktionsproduktes auftritt. Die Analyse der Gärungsprodukte ergab, daß - wie theoretisch vorauszusehen war - entsprechend einem Molekül abgefangenen Acetaldehyds ein Molekül Glycerin infolge Reduktion eines Zucker-Halbmoleküls gebildet wurde. Die Umsetzung bei Gegenwart sekundärer schwefligsaurer Salze verläuft demnach folgendermaßan:

$$
\mathrm{C}_{6} \mathrm{H}_{12} \mathrm{O}_{6}=\mathrm{CO}_{2}+\mathrm{CH}_{3} \mathrm{CHO}+\mathrm{C}_{3} \mathrm{H}_{8} \mathrm{O}_{3} \text {. }
$$

Diese Aufspaltung haben Neuberg und Reinfurth im Gegensatz zur GAY-LUSSACschen Normalgleichung als die zweite Vergärungsform bezeichnet. Die korrelative Beziehung zwischen Acetaldehyd und Glycerin war in jedem Momente vom Gärungsbeginn bis zum Ende durch die Konstanz ihres molekularen Verhältnisses analytisch nachzuweisen (NEUBEEG and HrRSCH). In gleicher Weise wie bei den schwefligsaueren Salzen trat in Gegenwart von Dimethylhydroresorcin die veränderte Umsetzung gemäß der zweiten Gärungsgleichung ein. Zwei Moleküle Dimethylhydroresorcin kondensieren sich unter Abspaltung eines Moleküls Wasser mit einem Molekül Acetaldehyd, während eine entsprechende Menge Glycerin als korrelatives Reduktionsprodukt gebildet wird ( $D i$ medonverfahren von NEUBERG und REINFURTH).

Aber nicht nur die als spezifische Carbonylreagenzien wirksamen sekundären schwefligsauren Salze und das Kondensationsmittel Dimethylhydroresorcin, sondern auch schwach alkalisch reagierende Carbonate, Bicarbonate, Phosphate usw. konnten eine Ausschaltung des Acetaldehyds aus dem normalen Gärverlauf erzielen. Wir haben schon oben erwähnt, welch wichtige Rolle der Cannizzaroschen Reaktion bei der Gärung zuzusprechen ist. Schafft man durch Alkalisierung mittels der genannten alkalischen Salze das für die optimale Wirksamkeit der Mutase günstige Milieu, so wird der intermediär auftretende Acetaldehyd nur in einem 'gewissen Umfange zu Äthylalkohol hydriert, im übrigen aber zu Essigsäure und Athylalkohol dismutiert. Dem dismutierten Acetaldehyd entsprechend entsteht in gleicher Weise, wie bei der zweiten Vergärungsform, eine äquivalente Menge Glycerin. Diese durch Mutasewirkung hervorgerufene Abänderung der Gärung wurde von NeUBeRG und Hirsch als dritte Vergärungsform bezeichnet und durch folgende Gleichung ausgedrückt:

$$
{ }_{2} \mathrm{C}_{6} \mathrm{H}_{12} \mathrm{O}_{6}+\mathrm{H}_{2} \mathrm{O}=\mathrm{CH}_{3} \cdot \mathrm{COOH}+\mathrm{C}_{2} \mathrm{H}_{5} \mathrm{OH}+2 \mathrm{C}_{3} \mathrm{H}_{8} \mathrm{O}_{3}+2 \mathrm{CO}_{2} \text {. }
$$

Bei der dritten Vergärungsform stehen Glycerin und Essigsäure im konstantem Verhältnis von 2. Molekülen: I Molekül. Auch diese Vergärungsform wurde in einem Umfange realisiert, der die quantitative Analyse aller Gärungsendprodukte in jedem Zeitpunkte des Abbaues gestattete. $\mathrm{Zu}$ erwähnen ist, daß neben der zweiten wie der dritten Vergärungsart, wenn auch in geringem Umfange, die normale (GAY-LUSSACsche) Zerlegung stattfindet. In eingehenden Untersuchungen (NeuberG, Hirsch und ReINFurTh) konnte gezeigt werden, $\mathrm{da} B$ in jedem Momente die verschiedenen Vergärungsformen mit der gleichen Reaktionsgeschwindigkeit nebeneinander verlaufen.

Bezüglich des Vorkommens der zweiten und dritten Vergärungsform in der Natur sei gesagt, daß sowohl Essigsäure als Glycerin (s. oben) stets in kleinen Mengen unter den Produkten der normalen Gäransätze aufgefunden werden konnten.

Die Bedeutung der Bren ztraubensäure bzw. des Acetaldehyds für die Gärung geht jedoch über die soeben besprochene Rolle als Zwischenprodukt beim Kohlenhydratabbau hinaus, indem der Acetaldehyd, wie alle hydrierbaren Substanzen, die alkoholische Gärung mächtig stimulieren. Solche Stimulatoren entstehen außer durch den Kohlenhyđratumsatz auch im Eiweißstoffwechsel der Hefezelle, da die Aminosäuren durch Desaminierung in die $\alpha$-Ketosäuren (O. Neubauer) und diese durch Abspaltung von Kohlensäure mittels Carboxylase (ebenso wie die Brenztraubensäure) in die entsprechenden um ein Kohlenstoffatom ärmeren Aldehyde übergeführt werden. In einer großen Reihe von Arbeiten haben NEUBerg und SANDBERG, von den Aldehyden und Ketonsäuren ausgehend, in den verschiedensten Substanzgruppen anorganischer und organischer Provenienz chemisch wohl charakterisierte Katalysatoren der Gärung nachweisen können. Diese Untersuchungen stehen im engsten Zusammenhange mit der Beobachtung, daß lebende Hefe sowie bemerkenswerterweise Hefefermentsaft imstande ist, stoffwechselfremde Substanzen - wie verschiedene Aldehyde, Ketone, Diketone, Nitrokörper, Hydroxylaminverbindungen, Nitrosoderivate, Disulfide, Thioaldehyde, reduzierbare Mineralstoffe - zu den entsprechenden Reduktionsstufen zu hydrieren; ein Vorgang, der insofern der Reduktion eines Zuckerhalbmoleküls (bei der 2. und 3. Vergärungsform) zu Glycerin entspricht, als hierbei anstatt des, ,inneren“ ein von außen herangeführter Wasserstoffacceptor in Funktion tritt. Das Gebiet der "phytochemischen Reduktionen" war durch seine enge Verknüpfung mit dem Gärungsvorgange von wesentlicher Bedeutung für die neueren Anschauungen über die Korrelation oxydativer und reduktiver Vorgänge in lebenden Zellen.

Die zentrale Stellung der Brenztraubensäure und des Acetaldehyds im Stoffumsatz der Hefezelle ging schlieBlich noch aus der experimentell erwiesenen Tatsache hervor, daß jene $Z$ wischenstufen des Abbaues gleichzeitig auch das Ausgangsmaterial für aufbauende Synthesen liefern. Diese biochemischen Synthesen kommen durch ein Ferment, die Carboligase (Neuberg und Hirsch) zustande, das die Fähigkeit besitzt, durch Verknüpfung zweier Aldehyde an ihren funktionellen Kohlenstoffatomen neue kohlenstoffreichere Verbindungen zu erzeugen. Bei der Vergärung von Zucker durch Hefe bzw. Hefefermentsaft in Gegenwa1 $t$ von Benzaldehyd konnte eine optisch aktive Substanz von der Zusammensetzung $\mathrm{C}_{6} \mathrm{H}_{5} \cdot \mathrm{CHOH} \cdot \mathrm{CO} \cdot \mathrm{CH}_{3}$ isoliert werden, die ihre Entstehung der Verknüpfung des beim Zuckerzerfall intermediär auftretenden Acetaldehyds mit dem zugesetzten Benzaldehyd verdankt. Bei der Umsetzung von Brenztraubensäure durch Hefe in Anwesenheit von Benzaldehyd wurde derselbe Ketonalkohol auf biochemischem Wege synthetisiert.

Die Aufklärung der intermediären Vorgänge beim Zuckerabbau durch die Hefezellen hat über das Gebiet der eigentlichen Gärungschemie hinaus eine prinzipielle Bedeutung gewonnen. Mit Hilfe des Abfangverfahrens gelang es bei den Kohlenhydratspaltungen durch die verschiedensten Mikroorganismen (NEuberG, Nord, WOLFF, CoHev) und durch atmende Kaltblütermuskulatur (HrRSCH) die gleichen $Z$ wischenprodukte $\mathrm{zu}$ isolieren. Das Bacterium coli, 
die Ruhrbacillen, die Erreger des Gasbrandes, die Essigsäurebakterien, die Schimmelpilze, bac. lactis aerogenes sowie überlebende Froschmuskulatur führen ihren Zuckerstoffwechsel durch die Zwischenstufe der Brenztraubensäure bzw. des Acetaldehyds hindurch; ebenso konnte nachgewiesen werden (NeUbeEG $u$. ArinsteIN), daß bei der Buttersäuresynthese durch die Butter- säurebakterien der Acetaldehyd bzw, die Brenztraubensäure in Form ihres Aldols die Zwischenstufe bilden.

Die dargestellten Ergebnisse sind von NeuBerg und seinen Mitarbeitern in der Biochemischen Zeitschrift, Band 3I-I26 (I9I I bis 1921 ), sowie in den Berichten der deutschen chemischen Gesellschaft I9II, I9I3, I9I4, I9I9, I920 veröffentlicht worden.

\section{EINZELREFERATE UND BUCHBESPRECHUNGEN.}

\section{ALLGEMEINES.}

O Die geschichtliche Entwicklung der Chemie. Von E. FÄRBER. Julius Springer, Betlin I921. XI, 3 I 2 S. u. 4 Taf. M. 78.- (M. 90.- ).

Das Buch ist, wie schon sein Titel sagt, keine Geschichte der Chemie im gewöhnlichen Sinn. FäRBER verfolgte offenbar bei der Abfassung seines Werkes den Plan, den Werdegang der Chemie als Teilproze $B$ wissenschaftlichen Denkens in die Entwicklung des menschlichen Geistes überhaupt einzuordnen. Dieser Versuch, die nackten Daten der Forschung und ihren experimentellen Inhalt von einem höheren, philosophisch begründeten Standpunkt zu überschauen, scheint mir restlos geglückt zu sein. In fesselnder Form rollt Verf. auf 300 Seiten das geschichtliche Bild der Chemie von den vorwissenschaftlichen Zeiten ab, durch Alchemie, Iatrochemie und Phlogistik hindurch bis zu den neuesten, auf der AvoGaDroschen Regel und der Thermodynamik fußenden chemischen und physikalisch-chemischen Theorien und Forschungsergebnissen vor uns auf. Gerade die Darstellung der modernsten Anschauungen wird hier besonders reizvoll, wenn man sie einmal in ihrem geschichtlichen Werden und ihrer historischen und logischen Bedingtheit gezeigt bekommt. Was die physiologische Chemie betrifft: um ein dem Mediziner naheliegendes Gebiet herauszugreif 2 , so ist es Verf. gelungen, die wechselnden Beziehungen und Grenzen zwischen Biochemie einerseits und ihren Nachbarreichen, der reinen Chemie auf der einen, der Biologie auf der anderen Seite, in wenigen, gedankenreichen Sätzen klar zu stellen (S. 29I). Jedem, der für chemische Probleme Interesse hat, kann das Buch auf das Wärmste empfohlen werden. Zum vollen Genuß des Werkes wird allerdings nur der kommen, der bereits über gewisse chemische Kenntnisse verfügt, etwa in dem Umfang, wie man sie bei jedem wissenschaftlich geschulten Arzte voraussetzen sollte. LAOUER (Frankfurt a. M.).

$O$ Der Gebrauch von Farben indicatoren. Ihre Anwendung in der Neutralisationsanalyse und bei der colorimetrischen Bestimmung der Wasserstoffionenkonzentration. Von J. M. KOLTHOFF. IV, I 44 S. u. I T. J. Springer, Berlin $192 \mathrm{r}$.

Zwei Methoden stehen uns zur Messung der Wasserstoffionenkonzentration zur Verfügung: die elektrometrische und die kolorimetrische, mittels Indikatoren. Die erstere erfordert jedoch eine komplizierte, kostspielige Apparatur, während die zweite, dank der Bestrebungen von Sörensen, Michaelis, Clark u. a., mit ganz einfachen Behelfen und mit geringer Mühe auszuführen ist. Jeder der sich über die Theorie der Indicatoren und ihre Verwendung orientieren will und $\mathrm{muB}$ - also alle Biologen, Serologen, wissenschaftlich arbeitende Mediziner - , wird in dem handlichen Büchlein des Verf.die erforderliche sachkundige Belehrung finden. Rona-Berlin.

\section{ANATOMIE UND ENTWICKLUNGSGESCHICHTE.}

O Leitfaden der topographischen Anatomie und ihrer Anwendung, für Studierende. Von O. OERTEL. S. Karger, Berlin 1922. IV, 226 S. M. 33.-

Das Buch behandelt die topographische Anatomie nach Körperabschnitten in übersichtlicher Gruppierung und leicht faßlicher Darstellung. Im Verhältnis zu dem kompendiösen Umfang ist der Inhalt sehr reichhaltig; zumeist sind alle wichtigen Tatsachen in klarer, knapper Form geschickt zusammengestellt. Doch leidet in einigen Kapiteln die Bearbeitung des umfangreichen Stoffes an dem Bemühen, allzusehr zusammenzudrängen. So ist die Darstellung der Gelenke etwas lückenhaft, läßt den topographischen Gesichtspunkt vermissen und beschränkt sich stellenweise auf eine Rekapitulation deskriptiver Systematik; bei den meisten Gelenken bleibt die Funktion und der topographische Beziehungswechsel in verschiedenen Bewegungsphasen ganz unberücksichtigt. Dic Zusammenfassung der Sprunggelenke zu einem zusammengesetzten Gelenk ist - sicherlich bei topographischer Betrachtung - verfehlt und irreführend; was ist ,die Fußgelenkkapsel“? Ein Mangel an Einheitlichkeit der Stoffbehandlung macht sich auch in einer stellenweise unexakten und wechselnden Nomenklatur bemerkbar. Schwerer wiegen wirkliche Fehler und Unklarheiten, wie sie sich in dem Abschnitt über das Bauchfell finden. Unrichtig sind die Angaben, daß die wandständige Fortsetzung des Mesocolon transv. die vordere Pankreasfläche bedeckt, daß die Milz sich im Lig. phrenico-gastricum entwickelt, daß ein Teil des Duodenum der Hinterwand des Netzbeutels angehört, daß die Radix mesent. die Aorta vor dem 4., die Vena cava aber vor dem 3. Lendenwirbel kreuzt u. a. Schwer verständlich ist die ausführliche fortlaufende Beschreibung gewisser Bauchfellblätter über viele Organe und Räume hinweg ohne vorherige Ubersicht über die räumlichen Anordnungen. Viel wichtiger wäre eine kurze Darlegung der den Situs bedingenden Entwicklungsvorgänge; aus ihrer völligen Ubbergehung ergeben sich manche Unklarheiten; m. E. ist ein Verständnis der Bauchfelltopographie ohne die Berücksichtigung primärer und sekundärer Fixation nicht zu erzielen. Weglassung bedeutet hier Erschwerung der Arbeit. Andere Abschnitte, besonders die den Kopf und Hals behandelnden, sind dem Verfasser besser gelungen. Während Genetisches, Physiologisches, Statisch-Mechanisches nurauch für einen Leitfaden - allzu vereinzelt herangezogen wird, ist in dem Buch treffend und einheitlich durchgeführt der Gesichtspunkt der Anwendung. Die in kurzen Schlagworten zahlreich eingestreuten, im Druck hervorgehobenen Hinweise auf Diagnostik, Pathologie und Therapie sind gut gewählt und fördern gerade durch die unmittelbare Einflechtung in den deskriptiven Text das Verständnis komplizierter Verhältnisse. Darstellungsweise und Inhalt dieser vielen zum Teil originellen Beispiele der Anwendung bilden den Hauptwert des Buches. Die 40 Abbildungen, die weder besonders instruktiv noch gut gezeichnet, außerdem durch allzu grobe Beschriftungslinien entstellt sind, wären entbehrlich.

VoGT (Würzburg).

\section{ALLGEMEINE PATHOLOGIE.}

Konstitution und Körperzustand. Von E. TOENNIES SEN. Münch. med Wochenschr. Jg. 68, Nr. 42, S. I 34 I. I92 I.

Verf. fügt zu den vererbten, also rein konstitutionellen Eigenschaften des Individuums und zu den erworbenen Somaschädigungen als drittes die konstitutionell-somatischen Eigenschaften hinzu, das sind diejenigen Erbfaktoren, die bei ihrer Entwicklung von den Reizen der Umwelt beeinflußt werden (physiologische Somavariationen). Die Konstitution ist etwas unabänderliches, aber die jeweilige absolute Größe der Leistungsfähigkeit wird innerhalb bestimmter Grenzen durch die Art der Beanspruchung verändert, der konstitutionelle Zustand wird verdeckt. Die Einzelheiten der Anschauung des Verfassers und ihre Begründung müssen im Original nachgelesen werden. RIISs. Familiäres Vorkommen von Cyclopie und Arrhinencephalie. Von A. KLOPSTOCK. (Pathol. Inst. des Auguste Victoria-Krkhs. Berlin-Schöneberg.) Monatsschr. f. Geburtsh. u. Gynäkol. Bd. 56, H. I/2, S. 59. $192 \mathrm{I}$.

Beschreibung von 2 Fällen von Cyclopie und Arrhinencephalie, die von dem gleichen, miteinander verwandten Elternpaar (Vetter und Base) stammten, im Abstand von einem Jahr geboren wurden. Uber die kausale Genese läßt sich nur sagen, daß sie zu suchen ist in einem in frühester Zeit der Keimentwicklung sich schon geltend machenden Vitium primae formationis, das erworben oder vererbt sein könnte. ZunTz.

\section{ALIGEMEINE THERAPIE.} THEORIE THERAPEUTISCHER WIRKUNGEN.

Der Mechanismus der herztonischen Wirkung kolloidalen Goldes. Von H. BUS QUET. Arch. internat. de pharmaco-dyn. et de thérap. Bd. 26, H. I $/ 2$, S. 75 . I 92 I.

Nach einer intravenösen Injektion von 0,OI g kolloidalem Gold ist nach einiger Zeit kein Gold im Serum des Versuchstieres mehr nachweisbar. Der Urin sowohl wie die Fäces, die innerhalb 48 St. nach der Injektion zur Ausscheidung kommen, enthalten gleichfalls kein Gold. (Grenzen der Nachweismethode nicht angegeben.) - Intravenöse Injektion von $0,003-0,005 \mathrm{~g}$ per $\mathrm{kg}$ Tier bzw. $0,002 \%$ Goldkolloid am isolierten Herzen von Hund und Kaninchen steigert die Herzaktion durch Vergrößerung der Amplituden. In situ macht sich gleichzeitig eine Verlangsamung der Herztätigkeit und eine mäßige Blutdrucksteigerung geltend. Im Gegensatz hierzu senkt sich nach Einspritzung löslicher Goldsalze $\left(\mathrm{AuCl}_{2}\right)$ von entsprechendem Goldgehalt der Blutdruck rasch, fast 